\title{
Computer Aided Business Model Design: Analysis of Key Features Adopted by Users
}

\author{
Boris Fritscher \\ Faculty of Business and Economics \\ University of Lausanne \\ boris.fritscher@unil.ch
}

\author{
Yves Pigneur \\ Faculty of Business and Economics \\ University of Lausanne \\ yves.pigneur@unil.ch
}

\begin{abstract}
Business model innovation comprises many theoretical models, frameworks and methodologies; however, there is still little in the way of digital support for them using Computer Aided Design software. In this paper, we present an analysis of real-world usage data from the first generation of such tools that were designed to support the business model canvas. We first present how two artifacts implement features such as colors for grouping, custom attributes and positioning of elements. An examination of how digital support brings about new opportunities in business modeling allows us to compare this with a paper-based version. We then analyze how variations of these features have been used in the real world. Finally, we examine the implications of our observations for the next generation of tools and the advancement of research for dedicated Computer Aided Design tools to support strategic objects such as business models.
\end{abstract}

\section{Introduction}

Growing competition between companies has led to the increasing popularity of business model innovation. Product innovation alone does not, however, enable companies to differentiate themselves enough to be able to compete [1]. Thus, businesses are finding that they need to look to methods and design techniques that can help them create new business models in order to outperform their competitors. To discuss business models, a common language is required.

Business model theories provide a common language by describing the components and the relationships that exist between them [2-5].

In order to help develop innovative ideas, it is not enough to provide an ontology that only includes the elements of a business model. The creation of a business model also requires a design process [6] that uses visual techniques to generate new ideas. Thus, the design process enables the transformation of business model concepts into paper-based canvases; these can then be worked on by practitioners.
The use of pen and paper adds a further set of constraints to a model's complexities. However, it can also provide creative freedom to "draw outside the box"; in other words, changes to the model can be made freely. Currently, the only common alternatives available to a leader of business model innovation are such generic tools as word processors, spreadsheet software and presentation programs. Generally, most models start out on paper as a series of boxes: these are later redrawn using presentation software, or are displayed as a list of bullet points.

Currently, there is a lack of consensus on which features should be included in a Computer Aided Design tool for strategy and how they can add value to the process. However, if we compare this with other design activities, there is certainly room for improvement. Looking at other design domains, advanced dedicated tools exist which can assist the designer throughout the design process. For example, architects and engineers have access to 3D Computer Aided Design (CAD) tools, which not only help them build a virtual prototype, but also simulate the structural integrity of their model. Programmers can use an Integrated Development Environment (IDE), which helps them develop software by providing content assist based on the context in which they are currently editing. Additionally, IDEs also provide pre-test run syntax checking to help produce valid code. In process management there are tools to design and simulate business model processes, which can then also be used as a dashboard for monitoring real-world processes.

By drawing parallels with these other domains we can describe what a Computer Aided Design tool for strategy, such as a business model, could look like. We distinguish between three major types of usage: a) creative design of the business model, b) assessing the designed business model, and c) managing the evolution of the business model and its variations.

The creative design of a business model heavily depends on the ability to choose the right level of abstraction for each component. In this situation, any tools have to be as non-constraining as possible in order to not hinder the creative effort and thus allow 
innovations to occur. Mostly, this results in the replication of a paper-based experience where everything is possible. The tool can also assist the user by constraining him to design within the chosen business model modeling language.

Digital formats enable the attachment of additional information relating to the elements, including: description fields, origin of sources, comments, category information, and grouping information. The visual appearance of a virtual element, such as color and form, can be altered at will without incurring the cost of recreating the element, as would be the case with a physical object. With the use of digital tools, it is also possible to quickly filter what users see and allow them to focus on a selection of available elements. This provides the capability to manage more information on one model.

In the context of a CAD tool for business models, we will explore the usage of three features: colors, attributes and positioning of elements.

Colors are a simple way to provide additional information; for example, labels can be used to group or connect elements.

Optional attributes, such as free form, guided or those used with formulas, can help in the storage of additional information in the model.

Element positioning can be free from constraints or can be assisted by the tool: it offers creative freedom and can help to reduce the burden of managing them.

Knowledge of the impact that the usage of these features has on the design of business models provides a basis on which new features can be built. In order to give an overview of potential extensions we will attempt to outline what these new features may look like.

In creative activities there is an opening phase during which any ideas are collected: this is followed by an exploratory phase and finally a closing phase, when a solution is arrived upon. For example, in brainstorming, ideas are grouped and combined [7]. In business modeling, this equates to verifying the coherence of a model, and whether or not any relationships between elements are defined. In this paper, we describe a dedicated computer assisted tool which has a knowledge of the meta-model of the modeling language. Thus, it can offer validation of constraints and best practice by automatically validating rules calculated on attributes which are then added to the elements.

When custom attributes are present, further opportunities are available. For example, users can add custom financial data and define relationships. This also allows users to perform calculations between them. Such calculations can build the basis for a simulation by asking what-if questions; for example, how the different estimates of market size, costs and revenues affect the potential profitability of the business model under design.

A business model evolves by adapting to its changing environment. It is therefore beneficial to be able to track the evolution of models, compare them and also simulate what-if questions at an environment level. Alternatives are generated by creating different business models for a multitude of scenarios [8]. Thus, innovative solutions are revealed by thinking outside of normal situations.

This paper's goal is to identify a set of features used in the design of a business model, such as color, examine changes brought about by different implementation choices and how they are used. It aims to compare these features in situations when alternative design tools are used and when traditional theory-based modeling methods are used. Based on the results, we then propose a set of recommendations for the next generation of business model design tools. Additionally, observing large-scale usage of a method also provides an opportunity for the co-evolution [9] of a tool and the theory that supports it. In this way, the theory itself is able to evolve and adapt to new use cases.

The structure of this paper follows design science recommendations [10]. In the next section we present the underlying theories on which our work is based. We then go on to describe our methodology. The artifact section that follows describes two instantiations of a tool to support business model design. The following two sections deal with the evaluation of the two artifacts and a discussion of the results. We conclude by discussing any implications of this study for future research.

\section{Justificatory knowledge}

Currently, there is no single unifying theory of business models; rather, there are silos of theories that lack consensus. However, the notion of the business model as a new unit of analysis is gaining in popularity and the field is moving towards conceptual consolidation [11].

One business model methodology in particular is starting to be widely adopted by practitioners: the Business Model Canvas (BMC). In the BMC, a business model is composed of nine building blocks, which describe it. These building blocks can be further grouped into four perspectives, as shown in Table 1. The main perspective is the offer (what we do), which connects the client perspective (who we do it for) and the activity perspective (how we do it). Finally, the financial perspective deals with profit (how much?).

Table 1 Business Model Canvas Components

\begin{tabular}{|l|l|l|}
\hline Perspective & Question & Building block \\
\hline Offer & What? & Value proposition \\
\hline
\end{tabular}




\begin{tabular}{|l|l|l|}
\hline $\begin{array}{l}\text { Client } \\
\text { (right side) }\end{array}$ & Who? & $\begin{array}{l}\text { Customer segment } \\
\text { Distribution channels } \\
\text { Customer relationships }\end{array}$ \\
\hline $\begin{array}{l}\text { Activity } \\
\text { (left side) }\end{array}$ & How? & $\begin{array}{l}\text { Key resources } \\
\text { Key activities } \\
\text { Key partnerships }\end{array}$ \\
\hline Financial & How much? & $\begin{array}{l}\text { Revenue stream } \\
\text { Cost structure }\end{array}$ \\
\hline
\end{tabular}

The positioning of these nine blocks is very important. Visually, they form separate groupings which help to structure the thought process and facilitate comparisons between the business models drawn using this method. As can be seen in Figure 1, the offer is in the center; to the right is the client perspective and revenue stream, whilst to the left is the activity perspective and cost structure.

The importance of having a visual canvas is anchored in the Design Thinking [12] process. This method is not a theory, but a set of practices popularized by companies such as IDEO to visualize and prototype concepts for the generation and validation of ideas.

The visual representation of the $\mathrm{BMC}$ has been downloaded over one million times by practitioners around the world since 2009 and has been used in creative sessions to innovate their business models.

Previous work [13] has shown that the BMC [14] is a good candidate for implementing a visual and accessible CAD tool for people who do not have an engineering background.

Other modeling tools do exist. Whilst these support business modeling, they differ from the simple visual interaction on which we focus.

Application-supported and object-oriented modeling, enterprise architecture modeling, or such generic tools as Visio and PowerPoint can all be used to draw up a model. However, they cannot be used to enforce modeling constraints - a requirement that we decided to include in this study.

Generic editors certainly exist, such as Protégée [15]; these are able to model and instantiate any domain that is supported by an ontology. This is a practical way to develop new meta-models or extend existing ones, although it is less practical for end-users.

Alternatively, a special editor can be generated, based on a Domain Specific Language (DSL) generated editor [16]. This allows model-specific constraints to be enforced.

Other methods have dedicated software; for example, SeamCAD [17] was specially designed to support the hierarchical natural of SEAM [2]. In addition, e3-value uses its own editor [18] to draw a custom representation. It also has some financial features, which allow it to export an Excel spreadsheet. However, both methods lack the visual simplicity and ease of use we want to achieve.
In this paper, we will describe two applications that implement the BMC. After presenting our methodology, we will analyze their use.

\section{Method}

In our study, we chose to follow a design science research (DSR) approach [10], [19] because our primary goal is to develop artifacts for the purpose of helping in the design of business models. Previously [13], we built an artifact to collect usage data on the features evaluated in this paper. Basing the evaluation on real-world usage data allowed us to show the relevance of the proposed ideas. Communicating this iteration contributed to knowledge in the form of a set of recommendations for future artifacts.

Analyzing the results was not straightforward. In order to help visualize and group the results, and thus obtain the relevant information, it was necessary to build artifacts using an iterative process.

\section{Artifact description}

In order to identify real-world use of features, we decided to analyze and compare usage data from two implementations of specialized business model design software made for the BMC. Both are web applications and include basic features, as well as those that are more advanced. However, they each take a different approach to how these features are implemented.

The first application, which we will refer to as $\mathrm{B}$, is an artifact that is the result of previous research [13]; however, it has not yet been evaluated in terms of features usage.

The second application, which we will refer to as $\mathrm{S}$, is an alpha version that was developed by a commercial entity using knowledge derived from research and guidance by the original authors of the BMC methodology.

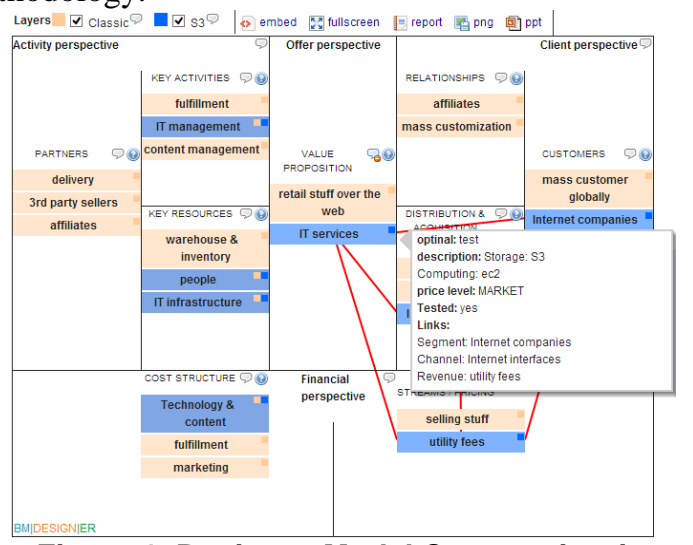

Figure 1. Business Model Canvas view in application B, with multiple colors, elements, links and attributes, and list positioning 
Both applications provide basic functionality by replicating the paper-based experience of the BMC. As can be seen in Figure 1 for B and Figure 2 for S, both display a visual canvas, with nine blocks making up a business model. Each of these blocks will receive elements that represent instances of the type of block.

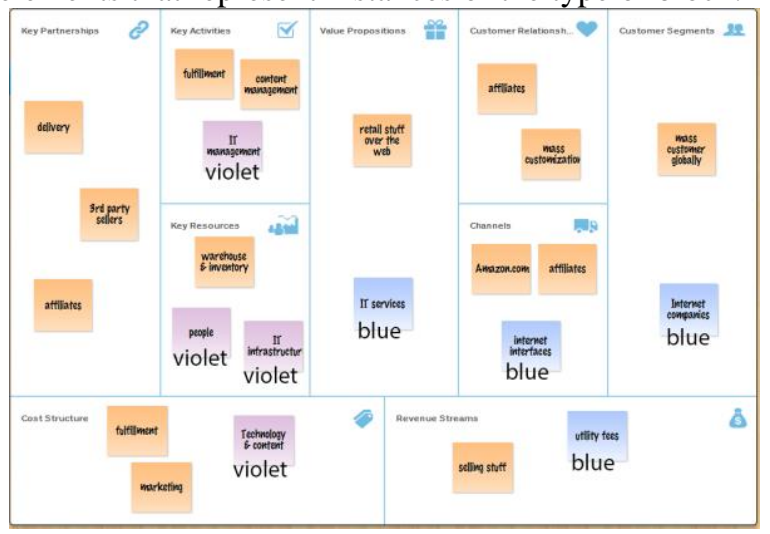

Figure 2. Business Model Canvas view in application S, elements with one color, free positioning

The elements can then be moved from one block to another or deleted as the design of the model evolves. The three functions on which the analysis of this paper is focused, and the different ways they are implemented by each application are summarized in Table 2 . The following sections explain the choices that underlie implementation and how they can be used in each artifact.

Table 2 Summary of differences between applications' functions

\begin{tabular}{|l|l|l|}
\hline Feature & B & S \\
\hline $\begin{array}{l}\text { Element } \\
\text { Colors }\end{array}$ & Has many colors & Has one color \\
\hline Attributes & $\begin{array}{l}\text { Optional, free } \\
\text { form and lists }\end{array}$ & $\begin{array}{l}\text { Optional free form } \\
\text { and financial with } \\
\text { benefits }\end{array}$ \\
\hline $\begin{array}{l}\text { Element } \\
\text { positioning }\end{array}$ & Guided, list & $\begin{array}{l}\text { Free inside each } \\
\text { block }\end{array}$ \\
\hline
\end{tabular}

\subsection{Element colors}

In application $S$ it was decided to replicate the paperbased experience as truthfully as possible. Thus, each element could have one of six colors, to equate with their real-world sticky note counterpart. On the other hand, for application B it was decided that color indicates some kind of belonging to a group of information. Utilizing the capabilities of a digital tool, an element was allowed to have multiple colors. The color of an element is given by the mix of colors that relate to each group it belongs to. Each individual color can be seen as a small square on the right of each element. The visibility of the elements that belong to each group can be toggled.
Users are free to use these colors in any way they like; no special indication is given in the software itself. A single color system can be replicated if the user chooses to limit himself to using one color at a time for each element. There are different ways of using colors to highlight elements. For example, a different color can indicate that an element is a comment, a future addition to the model or something that has been removed. A set of elements in a specific color can also be seen as an alternative model; this can assist in comparisons between multiple variations on the same canvas.

One of the best practices with the use of colors is to identify specific connected parts of the same business model. A multi-sided business model [20] has a common set of resources, which it uses to connect each multiple customer segment with a specific value proposition. For such cases, it is useful to pair the individual customer segments and their value propositions in their own color. Grouping can also be of use when highlighting new business opportunities, as can be seen in both Figure 1 and Figure 2: here, Amazon's standard business model is extended to offer a new value proposition of IT services to a new customer segment (blue) by reusing their existing infrastructure and IT knowledge. The main business model is presented using one color, whilst the new value proposition and customer segment are given in a different color. With regard to application B, Figure 1 illustrates the advantage of multiple colors where shared resources can be added to both color groups. It clearly illustrates that resources are used by both. On the other hand, in Figure 2, which shows application $\mathrm{S}$, an element can only have one color. The resource can use one of the two colors already used, but the information that they share is lost. Alternatively, a different color must be chosen (violet in the example) to indicate that something is shared. If there are more than two shared segments it still does not provide enough information to show the limitations of entirely replicating the paperbased environment.

\subsection{Attributes}

All the elements have one attribute in common: the name that is written and visible on them. Both applications allow extra content to be written on each element when accessing a detailed panel. In addition to this free form attribute, which is defined for each created element, application B provides attributes with a predefined selection of values for elements of specific blocks. These attributes and lists are taken from the original business model ontology [4]. By way of example, value proposition elements have a price level attribute that can have the following values: free, economy, market and high-end. To create a more 
semantic model, application B also allows the user to define his own attributes, title and value pairs. This gives more control over the structure of data than a single description field, but requires more work. All these additional attributes can be viewed by hovering over an element.

Application $\mathrm{S}$ does not provide custom attributes, but has specific predefined attributes on cost structure and revenue stream elements to allow the selection of basic predetermined profit calculation. In this paper, for the evaluation and comparison of attribute features we have only considered whether or not these calculation attributes are filled in; we have not sought to evaluate the efficiency of the calculation feature.

\subsection{Element position}

The extent to which the representation of a physical sticky note is replicated in both applications is different. In application $S$, the element represents a virtual sticky note that can be positioned anywhere in the block. On the other hand, application B positions elements in a list that can be reordered or moved from one block to another. However, they will always be aligned vertically, one above another. By allowing an element to take any position, it is possible to visually group them, although this does add to the task of managing their overlap and exact positioning. This can either be perceived as a positive or negative experience by the user. In list mode, the experience is closer to the bullet point list concept used in traditional office applications.

Application $\mathrm{S}$ has fixed size elements, which limits the length of text that can be displayed. In the list mode (B), the height of the item expands to fit all the text that is provided, as seen in the cost structure element "Technology \& content" in Figure 1.

\section{Evaluation}

The population of the real-world dataset, which we extracted from applications $\mathrm{B}$ and $\mathrm{S}$, is composed of over 5,000 business model enthusiasts from around the world. These include entrepreneurs, managers and consultants who have, for the most part, learned to use the BMC by reading the book that presents the method: Business Model Generation [14]. Since the applications are in English it is no surprise that the majority of users are from English-speaking countries. However, as can be seen in Table 3, other countries that have strong communities dedicated to Business Model Generation include Brazil, the Netherlands and Germany. For application B, the presence of China and France can be explained by the fact that a translation of the interface into these languages is available.
Table 3. Top countries listed by users

\begin{tabular}{|c|l|}
\hline B & $\begin{array}{l}\text { United States, Brazil, Germany, France, China, } \\
\text { Netherlands. }\end{array}$ \\
\hline S & $\begin{array}{l}\text { United States, Canada, Netherlands, Brazil, } \\
\text { United Kingdom, Germany }\end{array}$ \\
\hline
\end{tabular}

Business model data was extracted from the databases of the applications. After filtering this data 2,132 BMCs were collected using application B and the freely available research artifact over a period of 3 years. For application S, 4,921 BMCs were collected from alpha version subscribers over a one-year period.

The focus was on the use of more advanced features; thus, we based our selection only on what we considered to be valid canvases. Only canvases that contained between 9 and 60 elements were retained. Fewer than 9 elements means that the model cannot be complete, since there are 9 different blocks in the model. On the other hand, more than 60 elements means that there is a misuse of the model in relation to standard practice. Limiting the maximum number also eliminates those models in which users have chosen an incorrect abstraction level or have added too much detail on one canvas to that recommended.

In this section, we first analyze and compare color usage, before going on to look at attribute usage and the use of free element positioning. In order to compare color usage, BMCs were split into two groups: a) business models that only use one color, and b) business models that use more than one color.

Table 4. Percentage of business models with $x$ colors

\begin{tabular}{|l|r|r|}
\hline X colors & $\mathbf{B}$ (n=2132) & $\mathbf{S ~ ( n = 4 ' 9 2 1 )}$ \\
\hline 1 & $52.35 \%$ & $45.44 \%$ \\
\hline 2 & $19.84 \%$ & $15.40 \%$ \\
\hline 3 & $12.95 \%$ & $13.39 \%$ \\
\hline 4 & $7.08 \%$ & $10.40 \%$ \\
\hline 5 & $3.33 \%$ & $7.80 \%$ \\
\hline 6 & $1.45 \%$ & $7.56 \%$ \\
\hline
\end{tabular}

The first observation we can make from Table 4 is that colors were used in around $50 \%$ of canvases. This demonstrates some interest, although the figure should be higher if users really followed best practices. The somewhat higher number of multi-color canvases for models in application $\mathrm{S}$ can perhaps be explained by the user interface, which makes it easier to select different colors than in B where the color (representing a new group) has to be first added to the canvas before it can be used.

Single-color models are based on using the maximum number of complete business models; thus, elements should be shown in each of the nine blocks. Of greater interest is the use made of the grouping in multicolor BMCs because it allows us to understand whether or not the colors are used as shown in the theory: to split value propositions, customer segments and groups from each other. To better categorize the types of groups, we 
define a group type as a combination of the nine blocks which are either populated with an element in the group's color or not. This results in 512 (2 to the power of 9) possibilities.

\subsection{Business model canvases with a single color}

To compare single-color models with multi-color business models we merged all groups of multi-color business models into one, as if it was a single-color type group. If, as expected, coloring was used primarily to add additional information, the merging of business models should give similar results to their single-color counter parts in terms of completeness.

In Table 5 we list the most common types. As can be seen, over two-thirds of the business models were of the "complete" type, with elements in each block. The remainder showed the possible variations of the different types. In application B, for example, there were $341(66 \%)$ different types and in application $\mathrm{S}$ there were $475(92 \%)$. This may simply be due to the total number of canvases. The large number of complete $\mathrm{BMCs}$ is a good indication that the selection manages to include valid models.

Looking at the complete model (f), the top five mostoccurring types were the same for both applications and both types of canvases (single color, and multi-color). Use of the canvas without considering colors was identical for both applications. Furthermore, we can see that business models that do not contain financial data (nf) appeared twice as frequently in application B. This can be attributed to the fact that application $S$ provides several basic features related to profit calculation; thus, there is more incentive to fill out the financial elements.

The other three most popular business model types were shown to be business models that have one block left unfilled. The first one is where no customer relationship ( $r$ ) has been given and is consistent with our observations at workshops where users also had difficulties in filling in this block. Changes to BMC theory may help in making recommendations. It is also the least used block when considering total element percentages: $8 \%$ (Figure 3).

Business models with no partners (p) might be complete and valid, although it is unlikely in our connected world.

Business models without a cost structure (c) can be explained in terms of the user putting the cost information attributes directly onto elements in the other blocks; however, further analysis is required to confirm this proposition.

Based on observations from workshops and exercises carried out with students, we expected to find that there would be more models with only the right side (nl) filled out. In other words, we assumed that users would only fill in the client perspective of the BMC. However, this partial model usage seems limited to the group of users in this dataset.

Table 5. Most seen types for single color and merged multi-color canvases

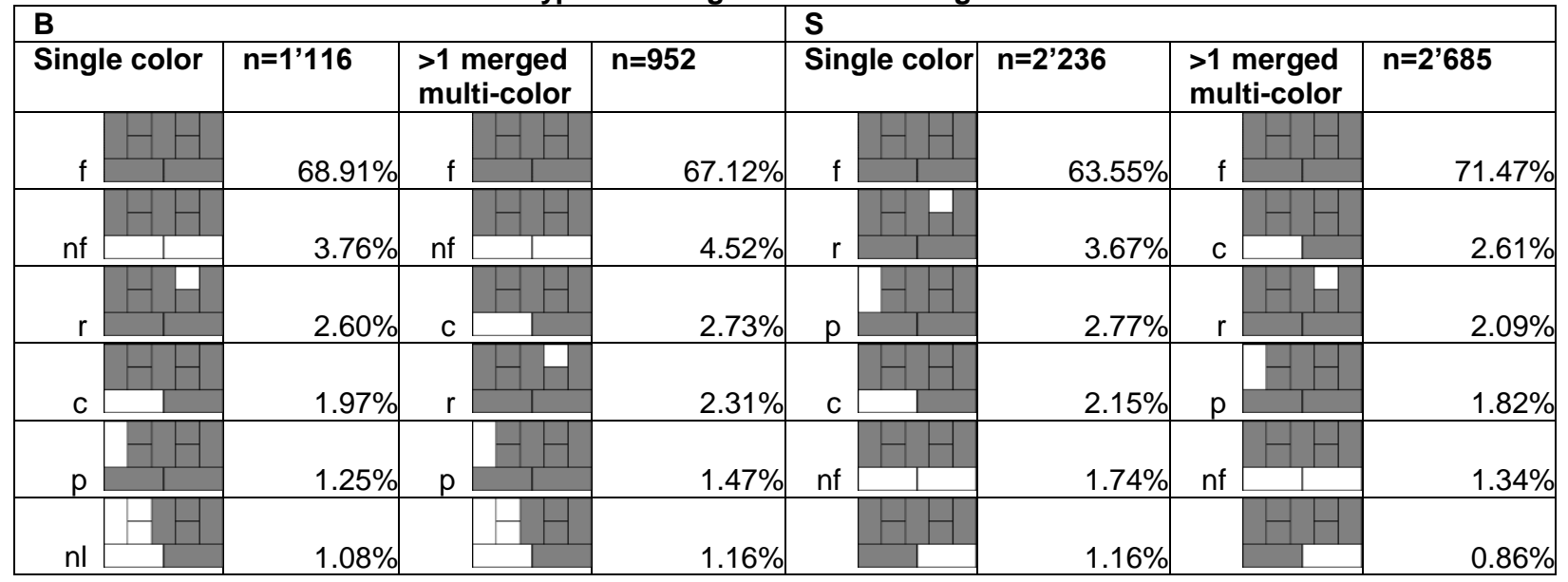

\subsection{Business model canvases with multiple colors}

Comparing multi-colored BMCs gave us an insight into how colors are used in each application. Unsurprisingly, the type that represents a full business model was the one most commonly found, as illustrated in Table 6. The percentages are lower than with a single-color model since, a business model is composed of multiple groups with different colors. Application B produced many more full models, which can be explained by the fact that any element can be in the color used as the main business model, 
and at the same time belong to additional color groups. With application S, however, there is only one color for each element. By changing its color, the element will be removed from the main color group and added to the new group; thus, it does not have a full group anymore.

Since many types of models resulted in low percentages, these were then further aggregated into four categories: full model, almost full, single block (only one block with the specific color), customer side (right part and eventually the value proposition).

Table 6. Percentage of types by artifacts

\begin{tabular}{|l|r|r|}
\hline Types & B (n=2'819) & S (n=9'693) \\
\hline Full model & $27.31 \%$ & $10.41 \%$ \\
\hline Almost full & $11.42 \%$ & $3.96 \%$ \\
\hline Single block & $7.52 \%$ & $24.77 \%$ \\
\hline Customer side & $4.15 \%$ & $16.13 \%$ \\
\hline
\end{tabular}

As presented in Table 6, large differences can be seen between the two applications. The reuse of elements in different colors with application B allows the right customer side to be connected with the resources on which it depends, which are on the left side. This may explain why there are fewer customer side types and more almost full types. From the point of view of application $\mathrm{S}$, there are more customer side types and fewer almost full ones.

Application B's multi-color ranking is almost identical to its single-color ranking, with no financial model type being the second most seen. Surprisingly, for application $\mathrm{S}$, the findings are completely different, with all nine possible types of single block groups ranking in the top most-found types.

The high number of single block types came as something of a surprise. It indicates that people only use one color for one block and that this does not relate to any business information used in the theory.

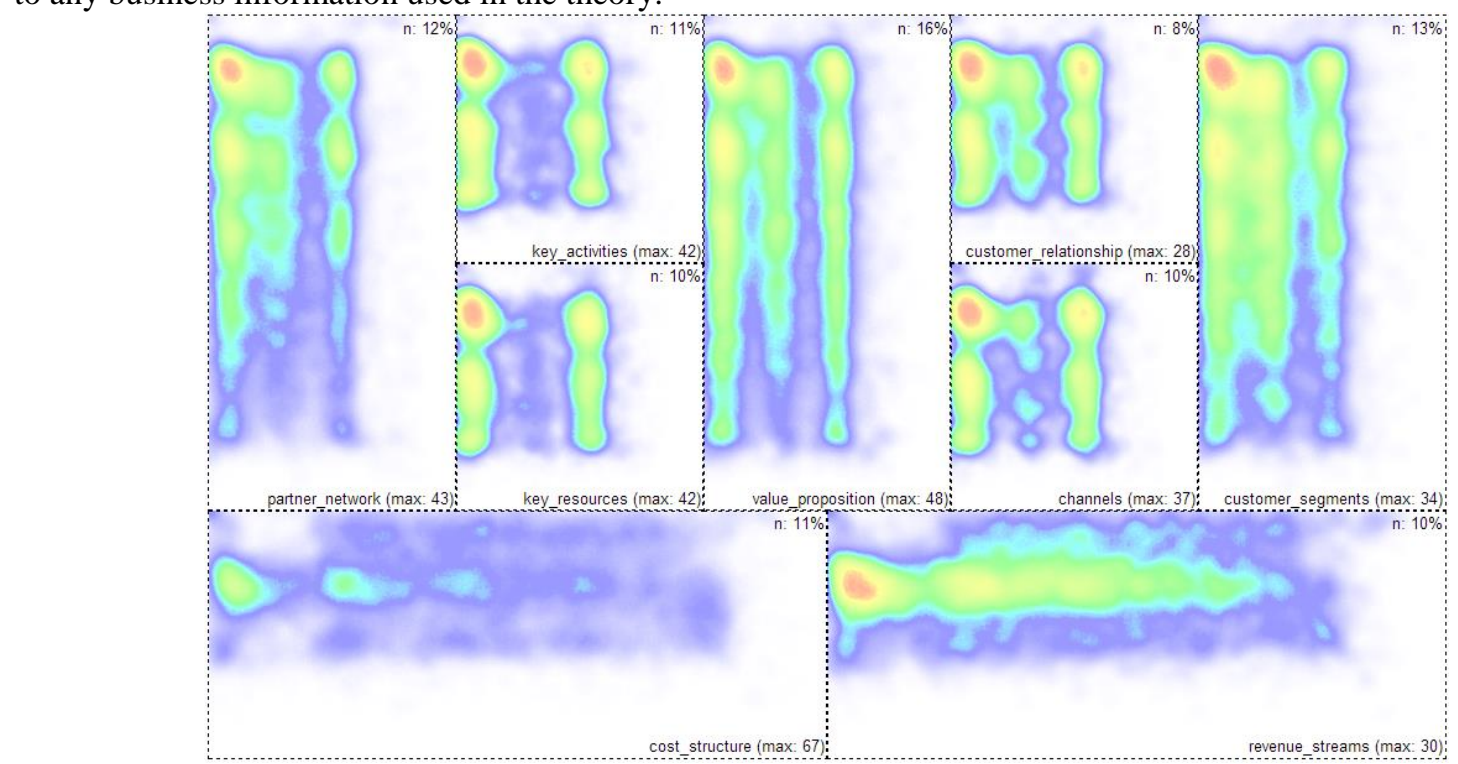

Figure 3. Heat map of element positions (top left corner) $n=163$ '589
In both models, left side and resource perspective types were almost non-existent even though they are illustrated in the pattern example given in the book that presents the method: Business Model Generation.

\subsection{Attribute usage}

On paper, BMC users had a tendency to add bullet points and sentences to explain their elements, rather than keywords. To check the usage of text in the applications, we calculated the word count for each element's name. The results are shown in Table 7. Even taking into account a different input system and different text size limits, the word counts were almost identical in both applications. In addition, $70 \%$ of the elements were found to have three words or fewer. Thus, the recommendation of using keywords has been followed.

Table 7. Percentage of elements with $\mathrm{x}$ words

\begin{tabular}{|r|r|r|}
\hline X words & $\mathbf{B}$ (n=65'490) & $\mathbf{S}$ (n=156'513) \\
\hline 1 & $27.94 \%$ & $30.85 \%$ \\
\hline 2 & $26.61 \%$ & $27.88 \%$ \\
\hline 3 & $17.50 \%$ & $16.51 \%$ \\
\hline 4 & $9.10 \%$ & $9.82 \%$ \\
\hline 5 & $6.12 \%$ & $6.28 \%$ \\
\hline 6 & $3.67 \%$ & $3.79 \%$ \\
\hline
\end{tabular}

Additional attributes were not often used: less than $10 \%$ of elements had descriptions, and less than $5 \%$ had a description that was longer than 15 words. Descriptions were mostly used to add small details such as sources.

Application B offered additional attributes in a selection list, but they were used in less than $1 \%$ of the cases. Custom attributes were used even less. 
Application S, on the other hand, offered calculation attributes, which we grouped into four categories. As shown in Table 8 they were used fully in one-third of all the business models.

Table 8. Percentage of financial attributes

\begin{tabular}{|l|r|}
\hline Type of financial attributes & S (n=4'921) \\
\hline No financials & $54.18 \%$ \\
\hline Only cost structure & $6.28 \%$ \\
\hline Only revenue stream & $6.64 \%$ \\
\hline Complete financials & $32.90 \%$ \\
\hline
\end{tabular}

\subsection{Element positions}

In application B, where there was free positioning of elements, users tended to align their elements in a grid format. We analyzed the position of each individual element by drawing a heat map to represent the density of the top left corner of each element. The result of the 163,589 elements can be seen in Figure 3.

For the horizontal blocks (cost structure and revenue stream), the elements were placed on a horizontal line and for the vertical blocks the elements were placed on a vertical line. Furthermore, three distinct vertical lines can be observed: the middle one is from cases where the user aligned his elements into a single center-column layout, while the two outer ones are from a two-column layout. The spacing between the columns matches the width of an element. For the vertical blocks it can also be seen that there are hotter points showing on the vertical line, spaced exactly by the height of an element.

In each block the hottest point is always in the top left corner, meaning that even for models with few elements, users tended to start adding the first elements to the top left rather than the center. To further verify the usage of a grid layout we computed the delta distance between the closest elements in the same BMC.

Table 9 shows that $50 \%$ of all the elements are aligned with their closest neighbor: same top position if in a horizontal block, same left position if in a vertical block, with a tolerance of $5 \%$.

Table 9. Elements matching a grid layout

\begin{tabular}{|l|r|r|}
\hline Block & $\begin{array}{l}\text { Elements } \\
\text { with same } \\
\text { top } \\
\text { position }\end{array}$ & $\begin{array}{l}\text { Elements } \\
\text { spaced by the } \\
\text { width of an } \\
\text { element }\end{array}$ \\
\hline Cost Structure & $51.71 \%$ & $40.13 \%$ \\
\hline Revenue Stream & $46.82 \%$ & $38.79 \%$ \\
\hline Block & $\begin{array}{l}\text { Elements } \\
\text { spaced by } \\
\text { the height } \\
\text { of an } \\
\text { element }\end{array}$ & $\begin{array}{l}\text { Elements with } \\
\text { same left } \\
\text { position }\end{array}$ \\
\hline Value proposition & $34.38 \%$ & $56.27 \%$ \\
\hline Customer Segment & $35.71 \%$ & $50.47 \%$ \\
\hline Partner Network & $37.92 \%$ & $51.03 \%$ \\
\hline
\end{tabular}

\section{Discussion}

This paper's goal is to acquire a comprehensive picture of how real-world use is made of such features as color, attributes and positioning in the current versions of business model design tools. To that end, we collected and evaluated data from two applications. Tests with students and workshops revealed signs that many users view the blocks of the BMC as individual check-lists to be filled out. Consequently, they did not go into detail about the relationship between the elements. According to our data, $50 \%$ of users did not use color to code their business model. However, business model completeness in single-color models and multi-color models did appear to be similar. Nonetheless, without the information provided by the grouping of the multi-color system, it is not possible for the tool to provide support for advanced model assessment and validation. Instead of using color grouping to link the elements, links between elements can be specified explicitly. This was tested in the case of application B, where links can be created by dragging and dropping one element onto another. The links can then be shown visually and connected elements are listed in a pop-up format, as shown in Figure 1. However, in the case of application B, link usage was nearly non-existent. It appears, therefore, that inferring information from colors is a better working system which is simple to use and an ideal compromise. To overcome the limitations of a singlecolor only system, a combination of systems may offer promise. For example, users could easily toggle a color on an element and then use these color associations in the system to extract the different groups and parts of a business model pattern, such as the double-sided business.

Proposition 1: A CAD tool for strategy should use simple visual indications, such as color tagging, to increase the semantic value of the model.

Contrary to the use of CAD tools in other domains, support tools aimed at the business level cannot (for now, at least) expect to be used by those who are specially trained in the software. Therefore, it is up to the tools themselves to offer users a more guided approach. As our results have shown, with the low usage of such custom features as attributes and advanced multi-color business models, it is not enough for features to be available and discoverable. Instead, they have to be promoted to the user, especially if there exists best practice in the use of such features. Simply giving an example of its use in theory does not seem to be directly transferable.

Disallowing the entry of multi-line text for element names did help in producing canvases that have mostly keyword entries. 
Proposition 2: A CAD tool for strategy should enforce or suggest good practice beyond implementing its underlying model.

The lack of use of custom attributes can have different explanations ranging from user interface problems and lack of choice in the list, to legal concerns relating to the sharing of confidential data. It seems most likely that a lack of access to the entered data from another view led to users being unable to see any benefit for its use. For attributes which offered a benefit, as was the case of financial data in application $\mathrm{S}$, usage was high; indeed, it was used in one-third of the business models.

This illustrates that, when an advanced feature provides sufficient added value, users are willing to invest in its use.

Proposition 3: A CAD tool for strategy should provide an incentive which justifies the need for providing additional information (e.g., numbers).

In terms of positioning, users had the opportunity to freely choose the elements' location in application $\mathrm{S}$. The majority of them positioned them in a grid layout. We can consider, therefore, that a large part of users did not use positioning to give additional information. As such, it would be possible for them to get by without having to make detailed decisions about the positioning of their elements.

Proposition 4: A CAD tool for strategy should support a beginner by simplifying his task and letting him focus on the core ideation process.

A few users recreated the multi-color systems in application $\mathrm{S}$ by manually stacking elements of different colors on top of each other with a small shift to give the appearance of multiple colors to the element on top of the stack. Whilst this works visually, it cannot be used by the system to process. Even then, it is cumbersome for the user because if an element is moved, additional colors have to be moved separately and then reordered to stack them correctly. Nonetheless, a system that is left open in this way offers many possibilities for working outside the box.

Proposition 5: A CAD tool for strategy should allow experts to create their own semantic meaning, such as by visually grouping elements through free positioning.

A compromise has to be found for the different phases: in the creative phase it should be more open, whilst in the assessments phase, some restrictions are necessary in order to apply validation rules.

\section{Future perspectives}

By considering these propositions and the need to move from design-supporting CAD tools for strategy to their next iteration, which allows for the assessment of business models, we are able to suggest potential improvements. In future, for example, simple rules could be validated which would make it possible to obtain better business models. Specifically, after a defined number of elements have been added, if there are still empty blocks, a wizard with trigger questions and explanations could be shown to the user, rather than the user having to look for himself. In the same way, if there is a complete model, but with only a single color, a wizard could suggest using the color system and show a tutorial which explains how colors can be used to group connected elements together. Another way to trigger thinking about the connected elements, which has been tested in a workshop, is to ask "trigger questions" which require users to think about multiple elements from different blocks to answer the question. With a support tool this could be combined with colors to tag the elements the user considers for his answer; these could then be validated against rules that are common to the trigger questions.

Promoting the collection of financial data is another topic which is open for improvement. In particular, the notion of cost could be made an attribute on each element and displayed as a summary in the cost structure. Digital tools have the capability to do this; however, it is not easy to replicate it in a dynamic way on paper. Thus, it is not accounted for in theory. These new possibilities will require the evolution of the BMC theory itself if it is to consider how more advanced financial computation could affect the different components.

Of particular interest is the co-evolution of the tool and the methodology that it implements. We can confirm that the customer relationship block is either of less importance than previously thought, or more likely that it needs to be more clearly defined in the methodology. This would bring an improvement to the BMC method based on information from the tool itself. An example can be given to illustrate such coevolution: when the methodology started using colors, these colors were picked up and interpreted as grouping when the tool was being engineered. This notion can be extended to the concept of layering in CAD software, which in turn has been integrated into the canvas methodology to visually show the evolution of one business model into another. Business model tools can extend their layering system to support this new concept and in turn come up with new additions to business model theory.

\section{Conclusion}

Inspired by design tools from other domains, we have sought to describe what computer aided design of business models could be like in the future. Guided by 
design science research, we have looked at the path to get there through the iteration of tools over design cycles. Starting with artifacts which replicate the paper-based experience of BMC methodology, we looked at the real-world usage of features which go beyond the basic 'sticky note experience' to include color, attributes and positioning.

Our data analysis has highlighted the importance of getting large-scale feedback beyond tests with students or experts. It also clearly showed the need to guide users as to the use of additional features and provide them with clear incentives to invest some time in learning them. We suggested some ways in which they could be implemented - for example, through the use of wizards and rules - although this needs further research and testing. Using the results of our study, the next iteration of tools can be better tailored to the use of practitioners.

Much research still needs to be done in order to bring the necessary improvements to Computer Aided Design Strategy software. As such, it will require strong collaboration between both strategy and IS researchers [21]. We see a high potential for the coevolution of tools and methodology, providing the opportunity to advance strategy theory as well as knowledge about IS support tools requirements and how these technologies will be adopted by users.

\section{References}

[1] D. J. Teece, "Business Models, Business Strategy and Innovation," Long Range Planning, vol. 43, no. 2-3, pp. 172-194, Apr. 2010.

[2] A. Wegmann, "On the Systemic Enterprise Architecture Methodology (SEAM)" Proceedings of the 5th International Conference on Enterprise Information Systems, pp. 483-490, 2003.

[3] J. Gordijn and H. Akkermans, "Value-based requirements engineering: exploring innovative ecommerce ideas," Requirements Engineering, vol. 8, no. 2, pp. 114-134, Jul. 2003.

[4] A. Osterwalder, "The Business Model Ontology-a proposition in a design science approach," Unil, 2004.

[5] H. Bouwman, H. Vos, and H. Timber, Mobile Service Innovation and Business Models. Springer, 2008.

[6] R. J. Boland and F. Collopy, Managing as designing. Stanford University Press, 2004.

[7] A. F. Osborn, Applied Imagination: Principles and Procedures of Creative Problem-Solving 3rd Edition, 3rd Rev. Creative Education Foundation, 1993.
[8] G. Ringland and P. P. SCHWARTZ, Scenario planning: Managing for the future. John Wiley \& Sons, 1998.

[9] P. Rittgen, "Success Factors of e-Collaboration in Business Process Modeling," in Advanced Information Systems Engineering, 2010, pp. 24-37.

[10] S. Gregor and A. Hevner, "Positioning and Presenting Design Science Research for Maximum Impact," MIS Quarterly, vol. 37, no. 2, pp. 337-355, 2013.

[11] C. Zott, R. H. Amit, and L. Massa, "The business model: Theoretical roots, recent developments, and future research,” papers.ssrn.com, vol. 3, no. September, 2010.

[12] R. Martin, "Design thinking: achieving insights via the 'knowledge funnel'," Strategy \& Leadership, vol. 38, no. 2, pp. 37-41, 2010.

[13] B. Fritscher, Y. Pigneur "Supporting Business Model Modelling: A Compromise between Creativity and Constraints," Task Models and Diagrams for User Interface, LNCS 5963, pp. 28-43, 2010.

[14] A. Osterwalder and Y. Pigneur, Business model generation : a handbook for visionaries, game changers, and challengers. Wiley, 2010.

[15] J. H. Gennari, M. a Musen, R. W. Fergerson, W. E. Grosso, M. Crubézy, H. Eriksson, N. F. Noy, and S. W. Tu, "The evolution of Protégé: an environment for knowledgebased systems development," International Journal of Human-Computer Studies, vol. 58, no. 1, pp. 89-123, Jan. 2003.

[16] M. Al Jallad, "REA Business Modeling Language: Toward a REA based Domain Specific Visual Language," KTH, 2012.

[17] L. Lê and A. Wegmann, "SeamCAD: object-oriented modeling tool for hierarchical systems in enterprise architecture," System Sciences, Proceedings of the 39th Annual Hawaii International Conference, 2006.

[18] J. Gordijn, H. Akkermans, A. Koks, and J. Schildwacht, "User manual e3-value editor.", Vrije Universiteit Amsterdam, pp. 30-35, Apr. 2004

[19] A. R. Hevner, "A Three Cycle View of Design Science Research," Information Systems, vol. 19, no. 2, pp. 87-92, 2007.

[20] T. Eisenmann, G. Parker, and M. Van Alstyne, "Strategies for two-sided markets," Harvard business review, Oct. 2006.

[21] A. Osterwalder and Y. Pigneur, "Designing Business Models and Similar Strategic Objects : The Contribution of IS Designing," Journal of the Association for Information Systems, vol. 14, no. 5, pp. 237-244, 2013. 